Copyright by the American Institute of Physics (AIP). Radiom, Milad; Robbins, Brian; Honig, Christopher D. F.; et al.,

"Rheology of fluids measured by correlation force spectroscopy," Rev. Sci. Instrum. 83, 043908 (2012); http:// dx.doi.org/10.1063/1.4704085

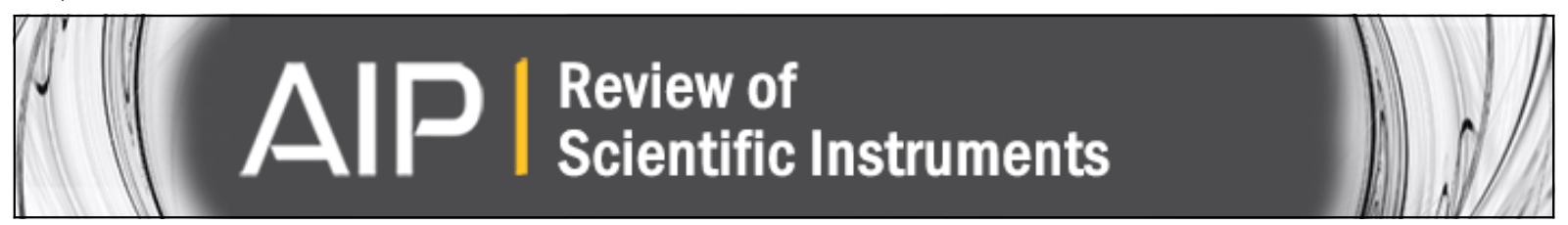

\title{
Rheology of fluids measured by correlation force spectroscopy
}

Milad Radiom, Brian Robbins, Christopher D. F. Honig, John Y. Walz, Mark R. Paul, and William A. Ducker

Citation: Review of Scientific Instruments 83, 043908 (2012); doi: 10.1063/1.4704085

View online: $\mathrm{http}: / / \mathrm{dx}$.doi.org/10.1063/1.4704085

View Table of Contents: http://scitation.aip.org/content/aip/journal/rsi/83/4?ver=pdfcov

Published by the AIP Publishing

\section{AlP Re-register for Table of Content Alerts}




\title{
Rheology of fluids measured by correlation force spectroscopy
}

\author{
Milad Radiom, ${ }^{1}$ Brian Robbins, ${ }^{2}$ Christopher D. F. Honig, ${ }^{1}$ John Y. Walz, ${ }^{1}$ \\ Mark R. Paul, ${ }^{2}$ and William A. Ducker ${ }^{1, a)}$ \\ ${ }^{1}$ Department of Chemical Engineering, Virginia Tech, Blacksburg, Virginia 24060, USA \\ ${ }^{2}$ Department of Mechanical Engineering, Virginia Tech, Blacksburg, Virginia 24060, USA
}

(Received 5 January 2012; accepted 1 April 2012; published online 23 April 2012)

\begin{abstract}
We describe a method, correlation force spectrometry (CFS), which characterizes fluids through measurement of the correlations between the thermally stimulated vibrations of two closely spaced micrometer-scale cantilevers in fluid. We discuss a major application: measurement of the rheological properties of fluids at high frequency and high spatial resolution. Use of CFS as a rheometer is validated by comparison between experimental data and finite element modeling of the deterministic ringdown of cantilevers using the known viscosity of fluids. The data can also be accurately fitted using a harmonic oscillator model, which can be used for rapid rheometric measurements after calibration. The method is non-invasive, uses a very small amount of fluid, and has no actively moving parts. It can also be used to analyze the rheology of complex fluids. We use CFS to show that (non-Newtonian) aqueous polyethylene oxide solution can be modeled approximately by incorporating an elastic spring between the cantilevers. (C) 2012 American Institute of Physics. [http://dx.doi.org/10.1063/1.4704085]
\end{abstract}

\section{INTRODUCTION}

The time response of individual and collective motions of molecules and particles can vary over many orders of magnitude, thereby introducing considerable complexity into the study of polymeric solutions and particulate suspensions. ${ }^{1}$ These complex fluids are found in thin films as lubricants, in separation processes such as chromatography and filtration, and in many personal products such as shaving cream and toothpaste. Complex fluids exhibit a non-trivial response to an applied strain that is neither Newtonian nor purely elastic. One of the most common techniques for measuring the rheological properties of materials is the traditional stresscontrolled or strain-controlled mechanical shear-rheometer. Although the technique is extremely versatile, it suffers from a number of limitations. For example, the instruments typically require several milliliters of sample, and the spacing between the opposing plates is of order $1 \mathrm{~cm}$. As a result, the measured response obtained is an average of the bulk response that does not provide information about the local dynamics of heterogeneous systems. ${ }^{2}$ Moreover, conventional rheometers have a relatively limited range of oscillation frequencies that can be accessed. Specifically, the finite mass of the device (i.e., parallel plate and cone-and-plate) sets an upper limit of frequencies to about $100 \mathrm{kHz}$.

Recently, microrheology techniques were developed that overcome many of the limitations of traditional rheometers. $^{3-8}$ The most common of these are the onepoint (or one-particle) $)^{9,10}$ and two-point (or two-particle) micro-rheometers. ${ }^{6}$ Although very successful both one-point and two-point microrheologies have limits of applicability that suggest the need for complementary techniques. The video tracking technique used to measure particle displacements is limited to frequencies less than $30 \mathrm{~Hz}$ (half the video frequency). ${ }^{11}$ Obtaining higher frequency response

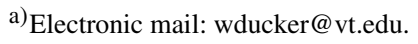

information has only been achieved using diffusing wave spectroscopy (DWS), which requires a concentrated particle suspension, or laser trapping of the two probe particles in conjunction with fast photodiodes. ${ }^{4,12,13}$ This can be a severe disadvantage for materials that have a broad range of characteristic time scales. The video tracking techniques typically can only resolve displacements of $\sim 20 \mathrm{~nm} .{ }^{14}$ The particleparticle separation distance in two-point microrheology must typically be of order $1 \mu \mathrm{m}$ to allow accurate resolution. The video techniques also require that particles are large enough to be visualized $(>0.5 \mu \mathrm{m})$. The technique is most effective for "soft" systems with elastic moduli $<100$ Pa. ${ }^{11}$

There are parallel efforts in microrheology measurements based on atomic force microscopy (AFM) cantilevers, ${ }^{15-18}$ following on recent success in understanding the dynamics of cantilevers in fluid. ${ }^{19-28}$ In the current work we make the extension from one cantilever rheology to two cantilever rheology, which we describe as correlation force spectroscopy (CFS). We are motivated to use two cantilevers rather than one for the same reasons that others used twoparticle rheology rather than one-particle microrheology. ${ }^{6,29}$ First, the correlation in fluctuations of two particles depends on the fluid structure between the particles, ${ }^{30}$ so two-particle microrheology has been used to examine different length scales of structure in the immersion fluid, such as chemically or physically cross-linked polymers, micellar solutions, and colloidal suspensions. ${ }^{30}$ By varying the separation between two cantilevers, we expect to be able to examine different length scales in solution. A second advantage of using two cantilevers instead of one cantilever is for singlemolecule force spectroscopy. The thermal noise, which limits resolution in force microscopy, is expected to be much smaller in the energy spectrum of the cross-correlation than in the auto-correlation. ${ }^{31,32}$ As a precursor to such singlemolecule studies, it is necessary to understand the correlations of the two cantilevers in fluid without the connecting single molecule. 
CFS is designed to conveniently measure the properties of a fluid on length scales of micrometers to nanometers and high-frequency range (500 kHz Nyquist frequency). Our device consists of a pair of micrometer-scale cantilevers that are closely spaced (see Fig. 1(a)). We do nothing to these cantilevers except observe the thermally excited vibrations of each cantilever using two laser beams (see Fig. 1(b)). The vibrations of the two cantilevers are not independent but are coupled through the fluid. Measurement and analysis of the correlations in motion can be used to quantify the nature of this coupling, and therefore to determine the properties of the intervening fluid. We can use cantilevers of various stiffness, so it is possible to measure the elastic response of materials with a large range of elastic moduli.

The principal disadvantage of CFS (in common with AFM and particle rheology) for rheology is that the shear stress at each frequency is not constant through the fluid. Exact solutions to the Navier-Stokes equation (for AFM) or unsteady Stokes equation (for one-point and two-point microrheology) can result in a precise knowledge of the dispersion of shear stresses through the fluid at each oscillation frequency of the micro-probe, but this is analytically cumbersome compared to traditional rheometry. Solutions are sometimes sought using finite element (FE) analysis. ${ }^{20,23,26,31,33}$

The current work is closely related to previous theoretical efforts to study the correlations between nanoscale and micron-scale cantilevers which have the advantage of high resonant frequencies and small spring constants. ${ }^{31,32}$ However, the overdamping of nanoscale cantilevers poses problems for deciphering the contributions to the dynamics from a tethered molecule. ${ }^{34}$ Here, we focus on commercially available $100-200-\mu \mathrm{m}$-scale cantilevers that are underdamped.

In this paper, we describe the instrument, and then compare experimental measurements to finite element analysis using theory developed by Paul and Cross. ${ }^{35}$ We then demonstrate that the instrument can resolve differences in the viscosity of Newtonian fluids, validating the use of the instrument as a rheometer. We present experimental measurements on a series of glycerol solutions and a complex fluid (1\% polyethylene oxide (PEO) solution) to demonstrate the capabilities of CFS at high frequency. We also use a model based on a simple harmonic oscillator (HO) to analyze the experimental data. The HO model can be used to quantify the magnitudes of the effective damping coefficient of the fluid surrounding each cantilever $\left(\gamma_{a}\right)$ and the effective damping coefficient of the fluid spanning the gap between the two cantilevers $\left(\gamma_{c}\right)$. Our results on the magnitudes of these parameters quantify the resolution of the device in the context of single-molecule force spectroscopy. ${ }^{32,34,36} \mathrm{We}$ propose a new scheme for molecule force spectroscopy using CFS.

\section{MATERIALS AND METHODS}

The apparatus consists of a pair of commercial AFM cantilevers (ORC8 types A or B, Bruker, CA) mounted in an antiparallel configuration between two glass slides, as shown schematically in Fig. 1. The cantilevers were positioned under an optical microscope and then glued in place.

Thermal drift is minimized because of small distances and material matching: the two cantilevers are joined by the cantilever chip $\left(\sim 3 \times 1 \mathrm{~mm}^{2}\right.$ of silicon nitride), a thin layer of glue, a glass slide, and a second cantilever chip. The cantilevers are mounted $>1 \mathrm{~mm}$ away from the glass slide, so there is minimal fluid coupling between the cantilever and rigid glass wall. In the vertical direction (Fig. 1(b)), all materials are matched for the left and right cantilever, and in the horizontal direction, the bases are separated by only about $400 \mu \mathrm{m}$ of glass, which is a much smaller connection than between tip and sample in a commercial AFM. There are no "moving" parts; the only motion is molecular motion of the fluid and the fluctuating deflection of the cantilevers due to interaction with the fluid at equilibrium.

The deflection of each of the cantilevers is measured by the light-lever technique, ${ }^{37}$ in which a laser (Schäfter + Kirchhoff GmbH, Hamburg, Germany) is reflected by the cantilever onto a position sensitive diode (Pacific Silicon
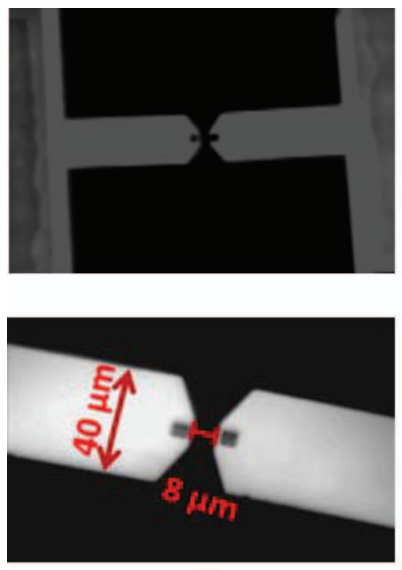

(a)

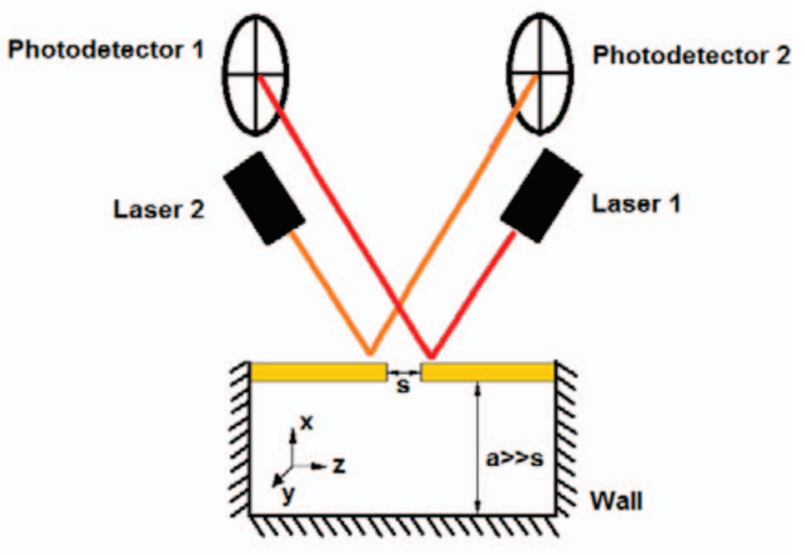

(b)

FIG. 1. (a) Light microscope images of closely spaced AFM cantilevers seen from above. The cantilevers are $100 \mu \mathrm{m}$ long and $40 \mu \mathrm{m}$ wide. The lateral separation between the cantilevers is $8 \mu \mathrm{m}$. (b) Schematic of the cantilevers and detection system in the CFS. $s$ is the gap between the two cantilevers and $a$ is separation from the bottom wall, which is $30 \mathrm{~mm}$ in our apparatus. 
Sensor, CA). In this case, we use a different wavelength (635 and $680 \mathrm{~nm}$ ) of laser for each cantilever, and (in some experiments) use a wavelength filter over each diode and different light paths to prevent cross talk between the signals. The signals are recorded synchronously by an Asylum Research Atomic Force Microscope controller (Nyquist frequency, $f_{\mathrm{Ny}}$ $=25 \mathrm{kHz}$ ) for type $\mathrm{B}$ cantilevers and a National Instrument (Irvine, CA) PCI-6110 Data Acquisition card $\left(f_{\mathrm{Ny}}=500 \mathrm{kHz}\right)$ for type A cantilevers. The fact that the device can be described in a couple of paragraphs gives an idea of its simplicity.

The phase lag between detection systems was shown to be negligible in an experiment when both lasers recorded the motion of a single cantilever. By measurement of the energy spectral density as a function of laser power, we found that the laser had a negligible effect on the temperature of the cantilever.

In each experiment, typically, we measure a time course of $5 \times 10^{6}$ voltage measurements from the diodes at $50 \mathrm{kHz}$ (for $100 \mathrm{~s}$ ) or $1 \mathrm{MHz}$ (for $5 \mathrm{~s}$ ). Subsequent data processing consists of: (1) dividing the whole data points into 10000 bins; (2) subtracting a linear curve fit from each bin to remove thermal drift in the signal; (3) taking a Fourier transform of each bin and calculating the power spectral density (PSD) for each cantilever averaged over all bins; (4) normalizing the PSD by dividing through by $\sqrt{A / f_{\mathrm{Ny}}}$, where $A$ is the area under the peak in the PSD. We normalize by $A$ because it is proportional to $k_{\mathrm{B}} T / k$, whereas the magnitudes in the PSD depend on the light lever gain, laser intensity, etc. ( $k_{\mathrm{B}}$ is the Boltzmann constant, $T$ is the temperature, and $k$ is the spring constant.) The normalized auto-correlation noise spectra (i.e., normalized power spectral density of each cantilever) are then $G_{11}(\omega)=\frac{1}{N} \sum_{\text {bins }} \hat{x}_{1}(\omega) \hat{x}_{1}^{*}(\omega)$ and $G_{22}(\omega)$ $=\frac{1}{N} \sum_{\text {bins }} \hat{x}_{2}(\omega) \hat{x}_{2}^{*}(\omega)$ and the normalized cross-correlation noise spectrum is $G_{12}(\omega)=\frac{1}{N} \sum_{\text {bins }} \hat{x}_{1}(\omega) \hat{x}_{2}^{*}(\omega)$, where $\hat{x}_{i}(\omega)$ is the Fourier transform of the normalized signal from each cantilever, $\omega$ is the frequency, and $N$ is the number of samples used in the average. The cross-correlation is normalized by the geometric mean of the normalization constant for each contributing signal. The auto- and cross-correlation functions are then the inverse Fourier transform of the noise spectra for which we used an inverse fast Fourier transform function: $\left\langle x_{i}(t) x_{j}(0)\right\rangle^{\prime}=\operatorname{IFFT}\left(G_{i j}\right)$, where $i=j$ for autocorrelation and $i \neq j$ for cross-correlation. The superscript on the correlation function $\langle\ldots\rangle^{\prime}$ denotes normalized calculations. Note that the normalization procedure described in step (4) gives a value of the auto-correlation of unity at zero time lag. We measure the spring constant of the cantilevers separately in an AFM by the thermal method. ${ }^{38}$

\section{MODELING OF THE STOCHASTIC DYNAMICS VIA DETERMINISTIC SIMULATIONS}

The stochastic dynamics of a cantilever pair in fluid was computed using deterministic calculations via the fluctuationdissipation theorem as described in Paul and Cross. ${ }^{30,34}$ This approach was recently validated by experiment. ${ }^{39}$ The autocorrelation, $\left\langle x_{1}(0) x_{1}(t)\right\rangle$, and cross-correlation, $\left\langle x_{1}(0) x_{2}(t)\right\rangle$, of equilibrium fluctuations in displacement of the free end of cantilever are proportional to the time course of the deterministic ring-down of the cantilevers due to the removal of a step force from the free end of one of the cantilevers:

$$
\begin{aligned}
& \left\langle x_{1}(0) x_{1}(t)\right\rangle=\frac{k_{\mathrm{B}} T}{F_{1}} X_{1}(t), \\
& \left\langle x_{1}(0) x_{2}(t)\right\rangle=\frac{k_{\mathrm{B}} T}{F_{1}} X_{2}(t),
\end{aligned}
$$

where $X_{1}(t)$ is the deterministic motion of cantilever 1 when the step force is removed from cantilever 1 , and $X_{2}(t)$ is the deterministic motion of cantilever 2 when the step force is removed from cantilever 1 . These equations are valid in the limit of small perturbations.

To compute the deterministic dynamics, we have numerically solved the time-dependent three-dimensional equations that govern the underlying fluid-solid interaction problem for two cantilevers using the finite-element-based ACE + Solver. ${ }^{40}$ We removed a step force from cantilever 1 and then simulated the deterministic ring-down of each cantilever. The ring-down of cantilever 1 was used to determine the auto-correlation of cantilever 1 (Eq. (1)) and the ring-down of cantilever 2 was used to determine the cross-correlation (Eq. (2)). In Sec. V, we also use a pair of coupled simple harmonic oscillators to model the deterministic dynamics.

\section{VALIDATION OF CFS AS A RHEOMETER FOR NEWTONIAN FLUIDS}

The auto-correlation and cross-correlation of the deflections of the cantilevers at $23^{\circ} \mathrm{C}$ are shown in Figs. 2 and 3 for three fluids of different viscosity: pentane $(\eta=0.22 \mathrm{mPa} \cdot \mathrm{s})$, water $(\eta=0.94 \mathrm{mPa} \cdot \mathrm{s})$, and $24 \mathrm{wt} \%$ glycerol in water $(\eta=2.07 \mathrm{mPa} \cdot \mathrm{s})$. Pentane was purchased from Alfa Aesar, water was Millipore purified, and glycerol was purchased from Sigma. The auto-correlation found using the cantilever pair is almost indistinguishable from the auto-correlation of an isolated cantilever. To validate the device as a rheometer, we compare the measured correlations to calculated values using the known viscosity of fluids. This calculation also depends on the density of the fluid, so this is a required parameter of the fluid.

Equations (1) and (2) allow us to model the stochastic fluctuations in deflection using deterministic simulations or calculations. For the validation of the rheometer, we use finite element numerical simulations, ${ }^{41}$ to calculate the ring-down $\left(X_{1}(t)\right.$ and $\left.X_{2}(t)\right)$ of the cantilevers for the geometries and conditions of the experiment. We used the nominal values of the cantilever geometry provided by the manufacturer, after using a light microscope to check that the dimensions are approximately correct. The end of the cantilever is tapered (see Fig. 1(a)), which we approximated with a stepped end (see inset of Figs. 2 and 3) for numerical simplicity. The values for the density $\left(4166 \mathrm{~kg} / \mathrm{m}^{3}\right)$ of the cantilever and its Young's modulus (140 GPa) were fitted to ensure that the theoretical values of the resonant frequency in air and the spring constant of each cantilever matched the corresponding experimentally measured values. This was done because of the large errors in 

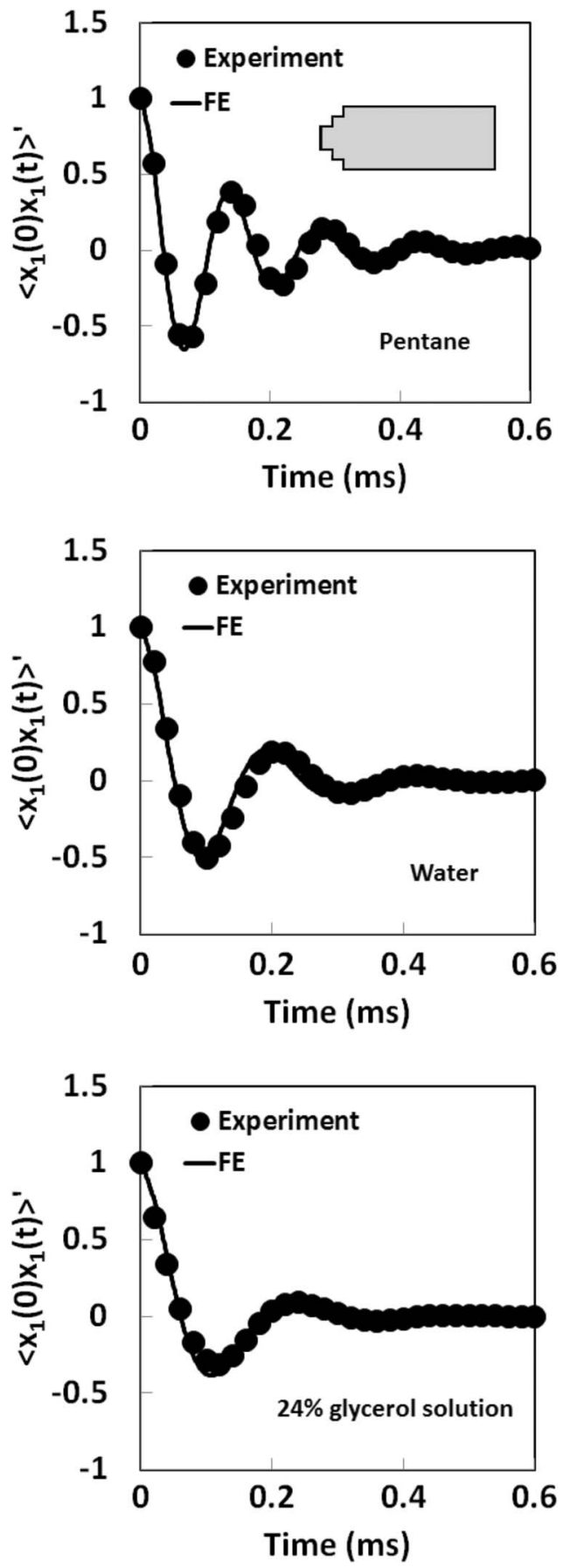

FIG. 2. Auto-correlation of equilibrium fluctuations in cantilever displacement in a series of Newtonian fluids. Experimental measurements at $23{ }^{\circ} \mathrm{C}$ are shown as data symbols reproduced from Ref. 39 and theoretical predictions are shown by the solid lines. Commercial AFM cantilevers are used for the experiments (ORC8 B: length $=200 \mu \mathrm{m}$, width $=40 \mu \mathrm{m}, k=0.1 \mathrm{~N} / \mathrm{m}$ ). The simulations used a cantilever with a stepped end as shown in the inset. The end is $10 \mu \mathrm{m}$ long and there is a single step on either side that is $4.5 \mu \mathrm{m}$ high and $7.5 \mu \mathrm{m}$ long to approximate the $59^{\circ}$ taper on the real cantilever. The prime on the left axis indicates that the correlation function is normalized.

determining precise values of the Young's modulus and cantilever thickness $(0.8 \pm 0.1 \mu \mathrm{m}$ from manufacturer).

The theoretical predictions of the cantilever auto- and cross-correlations using finite element numerical simulations are shown in Figs. 2 and 3. The theoretical predictions agree with experiment for different liquids of known viscosity. This demonstrates that the unknown viscosity of a test fluid could
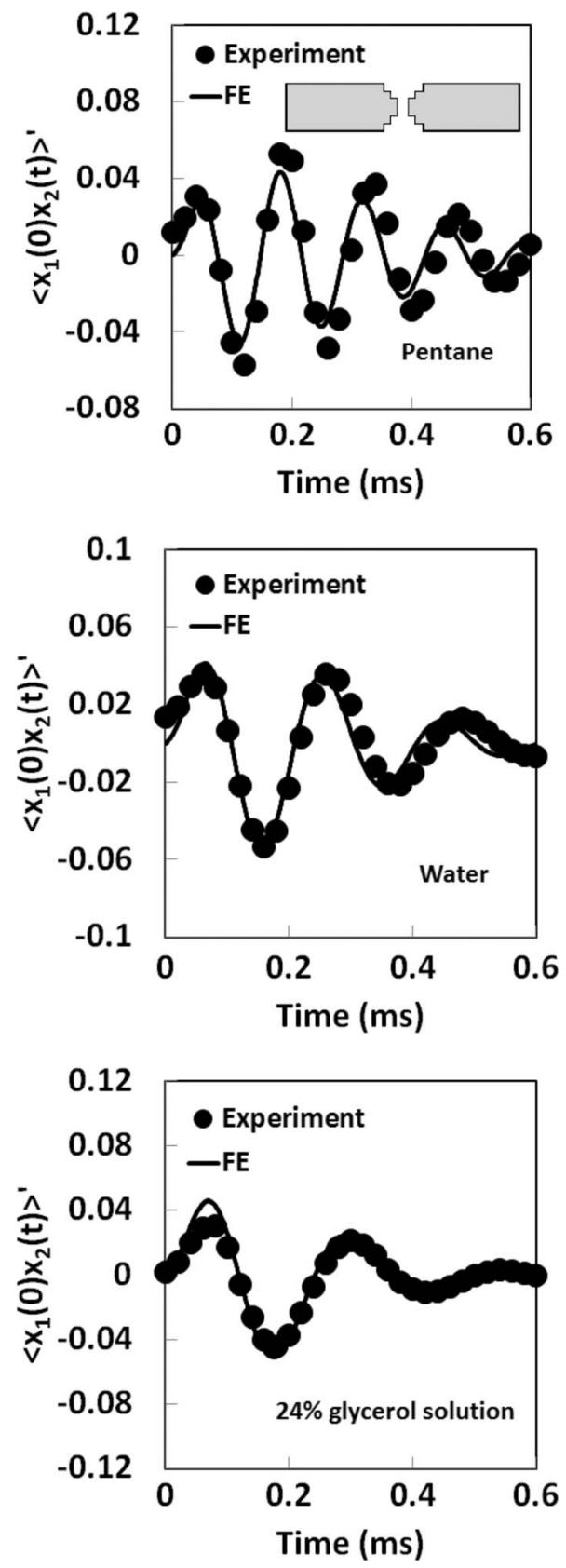

FIG. 3. Cross-correlation of equilibrium fluctuations in cantilever displacement for a pair of AFM cantilevers in a series of Newtonian fluids. Experimental measurements are shown as data symbols and theoretical predictions are shown by the solid lines. Commercial AFM cantilevers are used (ORC8 B: length $=200 \mu \mathrm{m}$, width $=40 \mu \mathrm{m}, k=0.1 \mathrm{~N} / \mathrm{m}$ ). Experiments were performed at $23{ }^{\circ} \mathrm{C}$. The cantilevers are separated by $8 \mu \mathrm{m}$. The prime on the left axis indicates normalized correlation function. Experimental data reproduced from Ref. 39

be extracted from measurements of either the auto-correlation of one cantilever or the cross-correlation between the cantilevers. The Fourier transform of the auto-correlation function (or in other words the single cantilever noise spectrum, $G_{11}$ ) has been used previously to measure the density and viscosity of Newtonian fluids by Boskovic et al. ${ }^{15}$ Our approach is different in that we include the frequency dependence of the dissipation when determining the noise spectrum $G_{11}{ }^{31}$ In the limit of high-quality oscillators, where the frequency 


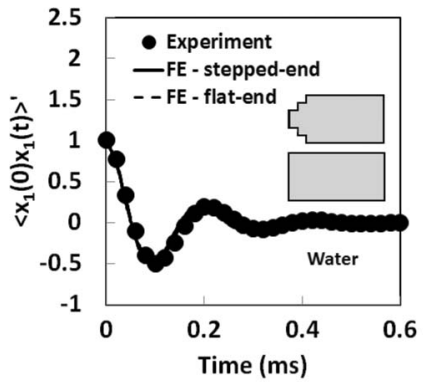

(a)

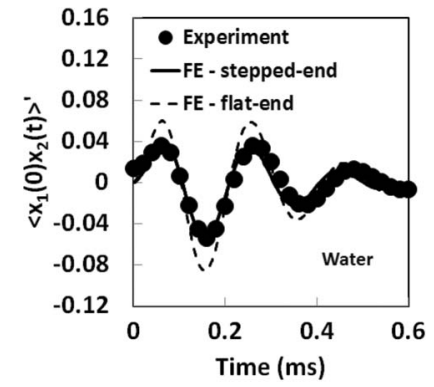

(b)
FIG. 4. Effect of geometry of unclamped end on simulation of (a) autocorrelation and (b) cross-correlation. Experimental points are shown for comparison. Note that the material properties are slightly different in the two simulations to allow fitting to the experimental resonant frequency in air The auto-correlation is largely unaffected (simulation lines in (a) overlay), whereas the magnitude of the cross-correlation is affected. Inset shows geometry of unclamped end.

variation of the fluid dissipation is negligible, the two approaches are equivalent.

One would reasonably expect that the cross-correlation would be dominated by the interaction between the closely separated ends of the cantilevers, and thus influenced by the shape of the unclamped ends of the cantilevers. To investigate this effect, we have also performed FE simulations on a flat-end cantilever. Figure 4 compares the FE simulations in water for a flat-end cantilever and the stepped-end cantilever (as shown in Figs. 2 and 3) to the experiment. The density and modulus fitted to the auto-correlation in air are slightly different to account for the change in mass of the cantilever when the shape is changed. The shape of the end of the cantilever has little effect on the magnitude of the auto-correlation in water, but there is a significant effect on the magnitude in the cross-correlation (Fig. 4). As expected, the presence of the extra material near the gaps between the cantilevers results in a larger magnitude of cross-correlation. This extra material would be an advantage in rheological studies. In contrast, coupling of the cantilevers via the fluid obscures the signal from single molecules straddling the gap, so a sharper unclamped end is an advantage in single-molecule studies.

\section{HO MODELING OF CANTILEVER RING-DOWN}

It is well known that a single cantilever in fluid can be represented as a damped simple harmonic oscillator. ${ }^{24,35,42}$ When the viscous dissipation is significant, the frequency dependence of the mass and damping terms must be included, ${ }^{31,35}$ but in the limit of small dissipation from the fluid, the added mass and the viscous damping can be considered frequency-independent, ${ }^{24}$ and one can evaluate the properties of the fluid at the reduced resonant frequency of the cantilever in fluid with a small amount of error.

We extend the idea of simple harmonic oscillator modeling to a cantilever pair in fluid. Each cantilever is replaced by a mass, spring, and damper, as shown in Fig. 5. The mass, $m$, is the effective mass of the cantilever plus the added mass due to the fluid motion. Each spring has a spring constant, $k$, and a damping coefficient, $\gamma_{a}$, that acts on a single cantilever

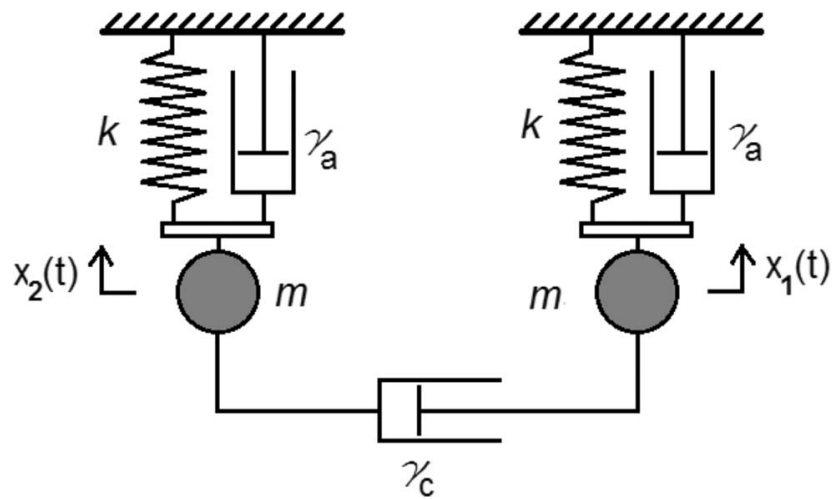

FIG. 5. Schematic of a cantilever pair modeled as a mass-spring-damper system. $m$ is the effective fluid loaded mass of the cantilever, $k$ is the spring constant, and $\gamma_{a}$ is the coefficient of fluid damping on an individual cantilever. $\gamma_{c}$ is the coefficient of fluid damping due to the fluid spanning the gap between the two cantilevers. The dashpot for $\gamma_{c}$ works such that when the left mass moves up the right mass is pulled down, and vice versa.

oscillating in fluid. (Here, we use two nominally identical cantilevers.) The damping due to the elastic material composing the cantilever has been neglected since it is much smaller than the viscous contributions. The coefficient of hydrodynamic damping of the fluid spanning the gap between the two cantilevers is $\gamma_{c}$. The plates shown in the schematic are massless. In general, $m$ and $\gamma_{a}$ are frequency-dependent parameters. We assume that they are constant ${ }^{24}$ and determine their numerical values by fitting to experimental data. Likewise we expect that $\gamma_{c}$ is a function of frequency, but here we also use a single numerical value by fitting to experimental data. We also expect that $\gamma_{c}$ is a function of cantilever separation, geometry, and configuration.

The equations of motion for the system shown in Fig. 4 are

$$
\begin{aligned}
& \ddot{X}_{1}+\left(\frac{\gamma_{a}}{m}+\frac{\gamma_{c}}{m}\right) \dot{X}_{1}+\frac{\gamma_{c}}{m} \dot{X}_{2}+\omega_{r}^{2} X_{1}=0, \\
& \ddot{X}_{2}+\left(\frac{\gamma_{a}}{m}+\frac{\gamma_{c}}{m}\right) \dot{X}_{2}+\frac{\gamma_{c}}{m} \dot{X}_{1}+\omega_{r}^{2} X_{2}=0,
\end{aligned}
$$

where $\omega_{r}^{2}=k / m$, and the quality factor $Q=m \omega_{r} / \gamma_{a} \cdot{ }^{31}$ For the initial conditions $X_{1}(0)=F_{1} / k, X_{2}(0)=0$, and $\dot{X}_{1}(0)$ $=\dot{X}_{2}(0)=0$, the solutions to Eq. (3) are

$$
\begin{aligned}
X_{1}= & \frac{\exp \left(-\omega_{d} t\right)}{2}\left[\cos \left(\sqrt{\omega_{r}^{2}-\omega_{d}^{2} t}\right)\right. \\
& \left.+\frac{\omega_{d}}{\sqrt{\omega_{r}^{2}-\omega_{d}^{2}}} \sin \left(\sqrt{\omega_{r}^{2}-\omega_{d}^{2}} t\right)\right] \\
& +\frac{\exp \left(-\frac{\gamma_{a}}{2 m} t\right)}{2}\left[\cos \left(\sqrt{\omega_{r}^{2}-\left(\frac{\gamma_{a}}{2 m}\right)^{2} t}\right)\right. \\
& \left.+\frac{\frac{\gamma_{a}}{2 m}}{\sqrt{\omega_{r}^{2}-\left(\frac{\gamma_{a}}{2 m}\right)^{2}}} \sin \left(\sqrt{\omega_{r}^{2}-\left(\frac{\gamma_{a}}{2 m}\right)^{2}} t\right)\right]
\end{aligned}
$$




$$
\begin{aligned}
X_{2}= & \frac{\exp \left(-\omega_{d} t\right)}{2}\left[\operatorname { c o s } \left(\sqrt{\left.\omega_{r}^{2}-\omega_{d}^{2} t\right)}\right.\right. \\
& +\frac{\omega_{d}}{\sqrt{\omega_{r}^{2}-\omega_{d}^{2}}} \sin \left(\sqrt{\left.\left.\omega_{r}^{2}-\omega_{d}^{2} t\right)\right]}\right. \\
& -\frac{\exp \left(-\frac{\gamma_{a}}{2 m} t\right)}{2}\left[\cos \left(\sqrt{\omega_{r}^{2}-\left(\frac{\gamma_{a}}{2 m}\right)^{2} t}\right)\right. \\
& \left.+\frac{\frac{\gamma_{a}}{2 m}}{\sqrt{\omega_{r}^{2}-\left(\frac{\gamma_{a}}{2 m}\right)^{2}}} \sin \left(\sqrt{\omega_{r}^{2}-\left(\frac{\gamma_{a}}{2 m}\right)^{2} t}\right)\right]
\end{aligned}
$$

where $\omega_{d}=\left(\frac{\gamma_{a}}{2 m}+\frac{\gamma_{c}}{m}\right)$. The initial displacement of cantilever $1, F_{1} / k$, scales all displacements, so Eqs. (4) and (5) are normalized by $F_{1} / k$.

Derivation of Eqs. (4) and (5) is provided in Appendix A. Appendix A also shows that these equations appear simpler if they are written in terms of the normal modes, $X_{s}=X_{1}$ $+X_{2}$ (symmetric deflection) and $X_{a}=X_{1}-X_{2}$ (antisymmetric deflection):

$$
\begin{gathered}
X_{s}=\exp \left(-\omega_{d} t\right)\left[\cos \left(\sqrt{\omega_{r}^{2}-\omega_{d}^{2}} t\right)\right. \\
\left.+\frac{\omega_{d}}{\sqrt{\omega_{r}^{2}-\omega_{d}^{2}}} \sin \left(\sqrt{\omega_{r}^{2}-\omega_{d}^{2} t}\right)\right], \\
X_{a}=\exp \left(-\frac{\gamma_{a}}{2 m} t\right)\left[\cos \left(\sqrt{\omega_{r}^{2}-\left(\frac{\gamma_{a}}{2 m}\right)^{2} t}\right)\right. \\
\left.+\frac{\frac{\gamma_{a}}{2 m}}{\sqrt{\omega_{r}^{2}-\left(\frac{\gamma_{a}}{2 m}\right)^{2}}} \sin \left(\sqrt{\omega_{r}^{2}-\left(\frac{\gamma_{a}}{2 m}\right)^{2} t}\right)\right],
\end{gathered}
$$

where the displacements are again normalized by $F_{1} / k$. We now observe that each mode is a decaying oscillation, and only the symmetric deflection depends on the viscous coupling between the cantilevers, $\gamma_{\mathrm{c}}$ (through $\omega_{\mathrm{d}}$ ): the antisymmetric stretch is the equation of motion for a single cantilever. This is intuitively obvious from Fig. 5 because the coupling damper is unaffected if one mass goes up and the other goes down. This viscous coupling speeds the decay of the symmetric mode and increases the period of the oscillation compared to a single cantilever.

We now need to obtain the parameters in the equation of motion for our particular experiment. The stiffness of each cantilever was measured in advance by Hutter's method, ${ }^{38}$ which is an analysis of the frequency spectrum of the autocorrelation of the isolated cantilever in air. In principle, $\gamma_{a}$ could be obtained from the frequency spectrum of the isolated cantilever in the appropriate fluid (similar to Hutter's method), but in practice, the parameters $\omega_{r}, \gamma_{a}$, and $\gamma_{c}$ were fitted to obtain the least sum of the normalized errors of autoand cross-correlation between the experiment with two cantilevers and Eqs. (4) and (5).The lumped mass of fluid for each cantilever was obtained from $m=k / \omega_{r}^{2}$. Because we neglected the frequency dependence of $\gamma_{c}, \gamma_{a}$, and $m$, we expect that the HO model will give better fits for fluids with a narrower resonant frequency, i.e., higher $Q$ factor.

Figure 6 shows the measured water and pentane data along with the fit using the HO model and the fluctuation dissipation theorem; the HO fit is good, it clearly captures the observed behavior. The fit parameters are shown in Table I. Comparing water to pentane, it is obvious that values of hydrodynamic damping coefficients as well as the fluid loaded mass are higher for a more viscous and denser fluid, so the model captures the essential features of the fluid. The fit in water (Figs. 6(c) and 6(d)) is good but not as good as for pentane (Figs. 6(a) and 6(b)). This is expected because the assumed frequency independence of mass and damping is a worse approximation for a more viscous fluid.

From the fit parameters, we have calculated other parameters such as the fluid loaded mass, $m$, and quality factor of the cantilever, $Q$, which are also shown in Table I. $\gamma_{a}$, theory was calculated from Eq. (29) in Ref. 31 for an infinite cylinder oscillating in fluid, ${ }^{24}$ whereas $\gamma_{a}$ is obtained for the actual geometry of the cantilever in the fluid including all tip effects and the interaction with the second cantilever. Nevertheless, the theoretical estimate is similar to the experimental value for pentane but about $50 \%$ larger for water.

The HO fit and FE simulation are compared in Figs. 6(a) and 6(b). Recall that the FE simulation uses only fitted values of modulus and density obtained from the power spectrum in air, whereas the HO model is fitted directly to the data in each fluid. The advantage of the $\mathrm{HO}$ model is that it can be obtained very quickly $(<1 \mathrm{~s})$ compared to the FE simulation ( $\sim$ week using a single workstation) and can be used to obtain intuitively useful modeling parameters such as $\gamma_{c}$.

\section{APPLICATION OF THE HARMONIC OSCILLATOR MODEL TO RHEOLOGY}

We now explore the use of CFS for rheometry for a model system consisting of a series of glycerol-water mixtures. The viscosity of the solutions was measured independently using a commercial rheometer (Anton Paar GmbH, Physica MCR 301 , Austria) and ranged from 1.5 to $7 \mathrm{mPa} \cdot \mathrm{s}$. The density remained approximately constant $\left(1075-1178 \mathrm{~kg} / \mathrm{m}^{3}\right)$ allowing us to isolate the effect of viscosity.

We measured the equilibrium fluctuations in cantilever displacement for a pair of cantilevers immersed in the series of glycerol-water solutions. These cantilevers had a higher resonant frequency and stiffness (ORC8 A, Bruker) than those used in the previous section, and were mounted as shown by light microscopy in Fig. 1(a). The experimental data are shown along with the results from the harmonic oscillator model in Fig. 7. Figure 7 shows that the harmonic oscillator model captures the essential features of the auto- and 


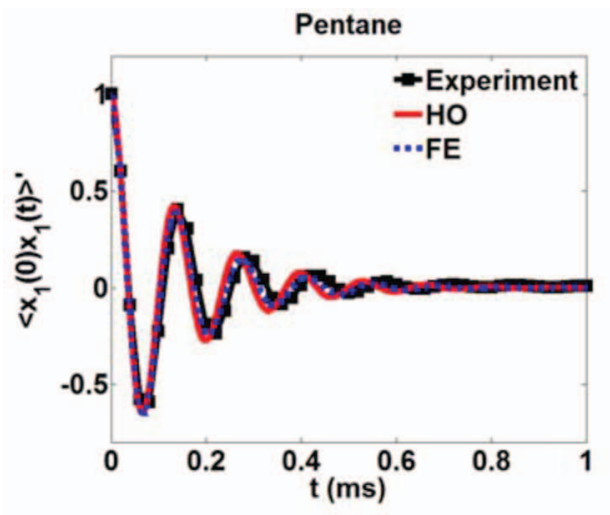

(a)

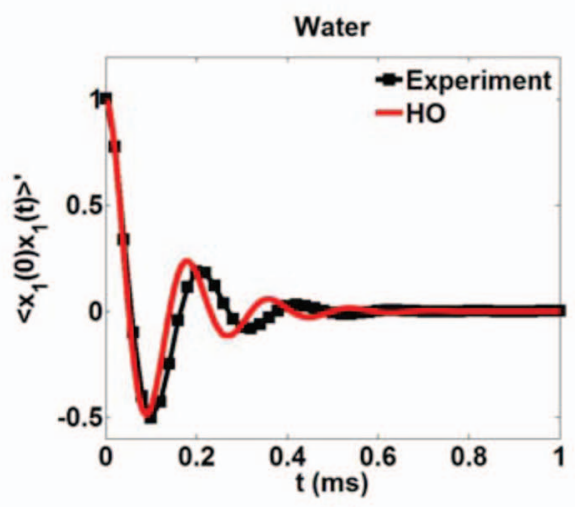

(c)

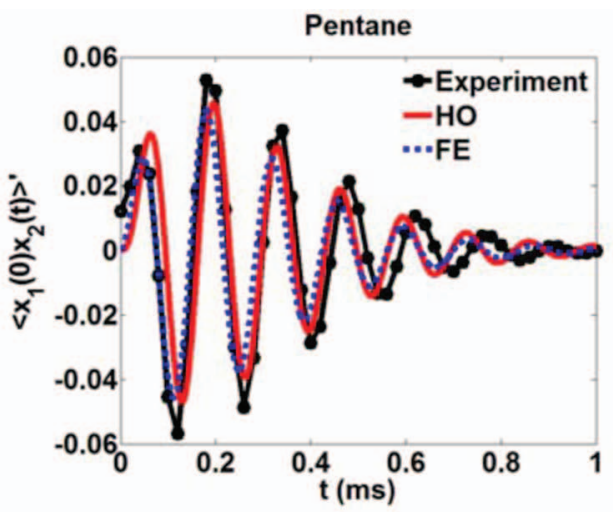

(b)

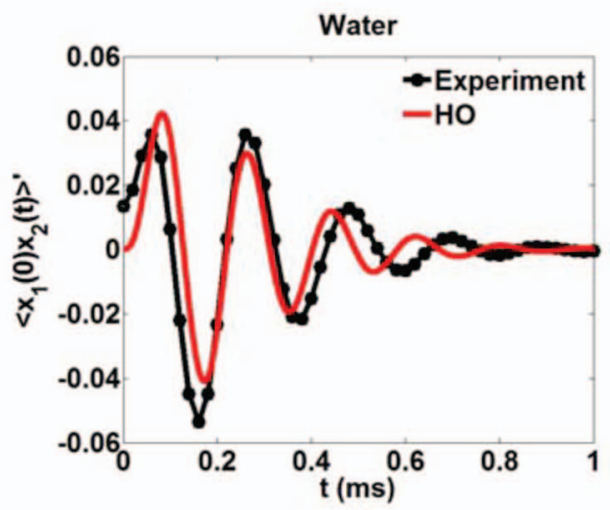

(d)

FIG. 6. Comparison of $\mathrm{HO}$ model with experimental data. (a) and (b) Auto- and cross-correlations in $n$-pentane. (c) and (d) Auto- and cross-correlations in water. Each plot shows the experimental results, the $\mathrm{HO}$ fit, and the FE model (using the known liquid viscosity and density). The ring-down from the HO and FE models has been used to yield the correlations in fluctuations using Eqs. (1) and (2). These results are for a pair of commercial AFM cantilevers (ORC8 B: length $=200 \mu \mathrm{m}$, width $=40 \mu \mathrm{m}, k=0.1 \mathrm{~N} / \mathrm{m}$ ) separated by $8 \mu \mathrm{m}$ at $23^{\circ} \mathrm{C}$.

cross-correlations; however, it is clear that the quality of the fit diminishes as the viscosity increases. Figure 8 shows $\gamma_{a}$ that has been extracted from the experimental data using the HO model and also the value predicted from theory for a single cantilever $\gamma_{a \text {,theory. }}{ }^{31}$ Each estimate of $\gamma_{a}$ shows a linear trend with viscosity (measured using Anton Paar $\mathrm{GmbH}$, Physica MCR 301, Austria). The value determined using the HO model gives smaller values of $\gamma_{a}$. Part of the reason for this discrepancy may be because the theory is for one cantilever and model and data contain a second cantilever. In addition, the theoretical value is for an infinite cylinder oscillating in fluid and neglects tip effects (that is $L / W \gg 1$ ) which is not the actual geometry in the experiment $(L / W=2.5)$. (For type B cantilevers where $L / W=5$, theoretical and experimental $\gamma_{a}$ agree much better as shown in Table I.) The important point is that $\gamma_{a}$ is linear in viscosity, which enables the HO model to be used to determine the unknown viscosity of a solution, provided that two calibration solutions of known viscosity are tested first to establish a calibration factor.

The damping coefficient due to hydrodynamic effects from fluid in the gap $\left(\gamma_{c}\right)$ is also linear in viscosity (Fig. 9). Again, this means that, given calibration data from solutions of known viscosity, the viscosity of unknown solutions can be measured. In contrast, the theoretical predictions using finite element simulations does not require calibration, and is predictive, but takes longer and does not directly produce intuitive parameters such as the damping coefficient. Table II shows values of the best-fit parameters of the HO model. Again, $\gamma_{c}$ is about an order of magnitude smaller than $\gamma_{a}$.

TABLE I. Fit parameters for HO model of the two cantilever system (Eq. (3)). $\gamma_{a}$, theory is calculated from Eq. (29) in Ref. 31. $m_{e}$ is the effective cantilever mass determined from measurements made in air using $m_{e}=k / \omega_{o}^{2}$, where $\omega_{o}$ is the resonant frequency in air and $m$ is the fluid loaded mass. Viscosity, $\eta$, was measured using a traditional rheometer.

\begin{tabular}{|c|c|c|c|c|c|c|c|c|}
\hline \multirow[b]{2}{*}{ Fluids } & \multicolumn{3}{|c|}{ Fit parameters } & \multicolumn{3}{|c|}{ Derived quantities } & \multirow[b]{2}{*}{$\gamma_{a, \text { theory }}[\mathrm{mg} / \mathrm{s}]$} & \multirow[b]{2}{*}{$\eta[\mathrm{mPa} \cdot \mathrm{s}]$} \\
\hline & $\gamma_{a}[\mathrm{mg} / \mathrm{s}]$ & $\gamma_{c}[\mathrm{mg} / \mathrm{s}]$ & $\omega_{r} / \omega_{0}$ & $m$ [ng] & $m / m_{e}$ & $Q$ & & \\
\hline Pentane & 0.51 & 0.077 & 0.38 & 44 & 2.4 & 4.1 & 0.58 & 0.22 \\
\hline Water & 1.1 & 0.14 & 0.28 & 79 & 4.6 & 2.5 & 1.62 & 0.94 \\
\hline
\end{tabular}



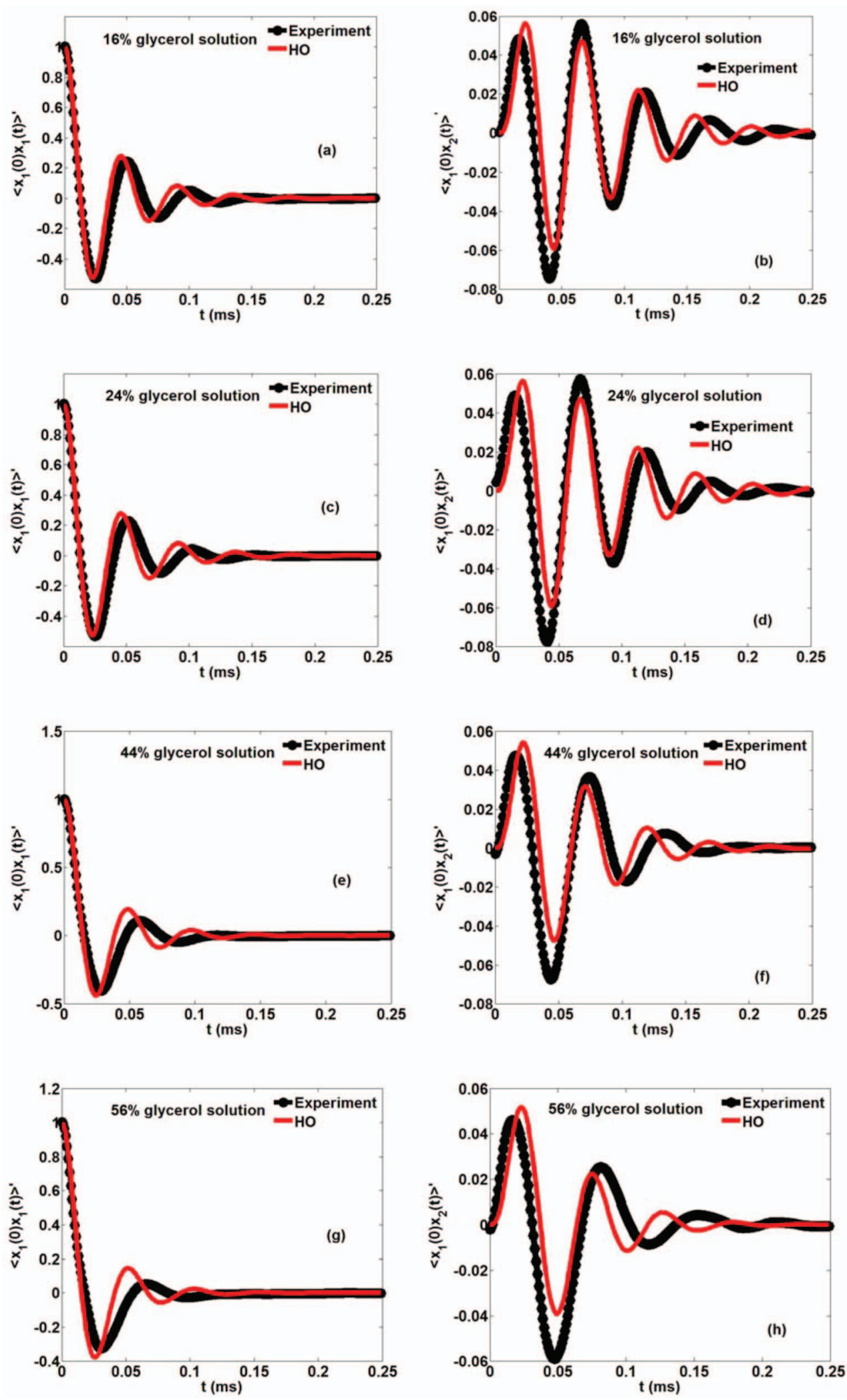

FIG. 7. Comparison of $\mathrm{HO}$ model with experimental data for the thermal vibration of two AFM cantilevers separated by $8 \mu \mathrm{m}$ at $23{ }^{\circ} \mathrm{C}$ in a series of glycerol solutions. Auto-correlation (a), (c), (e), and (g) and cross-correlation (b), (d), (f), and (h) of equilibrium fluctuations in cantilever displacement for a pair of AFM cantilevers (ORC8 A: length $=100 \mu \mathrm{m}$, width $=40 \mu \mathrm{m}, k=0.71 \mathrm{~N} / \mathrm{m}$ ). 


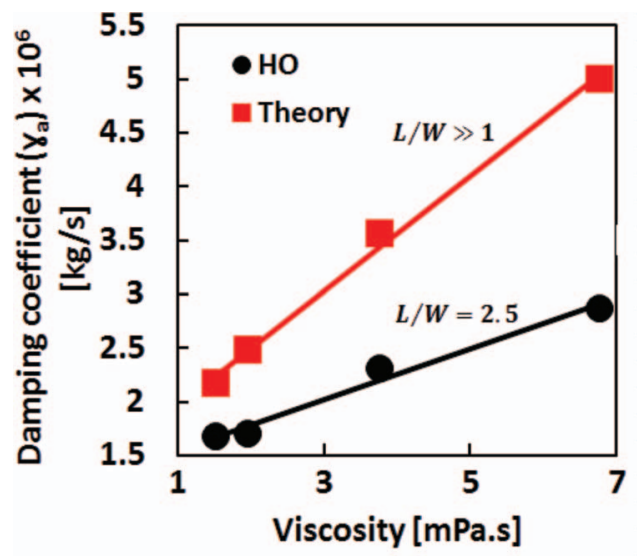

FIG. 8. Damping coefficient $\gamma_{a}$ as a function of viscosity measured by a conventional viscometer. Black circles are fitted damping coefficients from HO model of CFS data (Fig. 7). Red squares are theoretical damping coefficients, $\gamma_{a \text {, theory }}$, calculated from Eq. (29) in Ref. 31 at the experimentally measured resonant frequency. The solid lines are linear curve fits through the data points.

\section{APPLICATION OF THE HARMONIC OSCILLATOR MODEL TO RHEOLOGY OF NON-NEWTONIAN FLUIDS}

CFS can also be used to measure the rheological properties of non-Newtonian fluids at high resonant frequencies. We have measured the auto- and cross-correlation in the thermal vibrations of cantilevers immersed in $1 \%$ PEO (Aldrich, molecular weight $\left.=5 \times 10^{6}\right)$ (Fig. 10). The analysis of PEO data starts with applying an appropriate low pass filter to cantilevers fluctuation data in order to exclude correlations from the second and higher resonant frequencies. PEO is known to be shear thinning, which we confirmed by conventional rheometry for frequencies up to $100 \mathrm{~Hz}$ (see Fig. 11). Our objective is to understand the rheology at higher frequencies so we extrapolated a power law fit to the data to estimate the viscosity in the range of $2-64 \mathrm{kHz}$, which encompasses the resonance peak for the cantilever in 1\% PEO (Fig. 10(b)). For comparison, we also measured the fluctuations in a Newtonian solution, $56 \%$ glycerol in water, with a viscosity $6.77 \mathrm{mPa} \cdot \mathrm{s}$, that is in the extrapolated range of viscosities of $1 \%$ PEO near the resonance peak.

In this section, we use our HO model to extract the relevant fluid properties from experimental data. This provides a qualitative description of the data and physical insights into the rheometry on non-Newtonian fluids. Our interpretation of the fluctuations measured in $1 \%$ PEO via Eqs. (1) and (2) is constrained by the assumption of small perturbations that can be accurately approximated as linear functions of the disturbance - unfortunately, a generalization of the theoretical

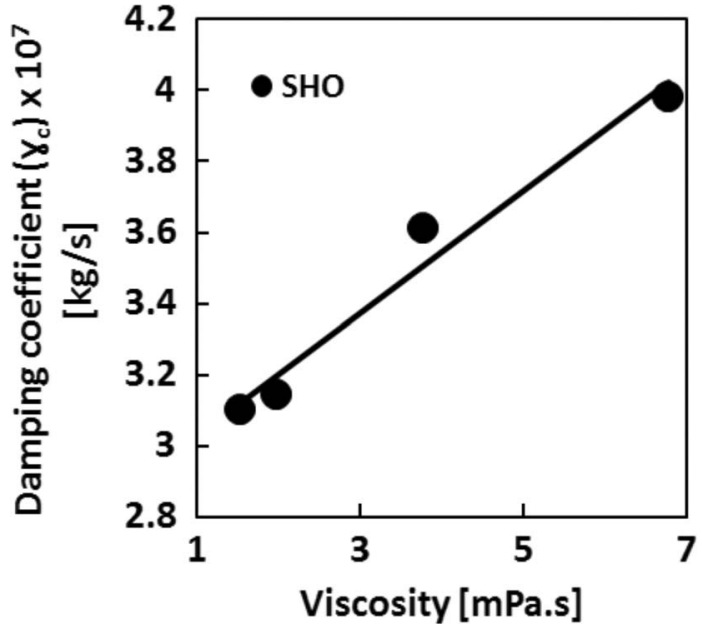

FIG. 9. Damping coefficient $\gamma_{c}$ as a function of viscosity that has been measured by a conventional viscometer. Black circles are fitted damping coefficients, $\gamma_{c}$, from HO model of CFS data (Fig. 7) and the solid line is a linear curve fit through the data.

approach using the fluctuation-dissipation theorem for nonNewtonian fluids is not currently available. We will maintain self-consistency by using an HO model with a linear response, but acknowledge that non-Newtonian fluids may be more complex.

Figure 10 shows the cross-correlation measured using CFS. We note the following qualitative observations:

(1) The auto-correlation (Fig. 10(a)) and noise spectrum (Fig. 10(b)) of a single cantilever immersed in 1\% PEO do not exhibit any obvious features that distinguish them from data in glycerol solution.

(2) The cross-correlation noise spectrum in 1\% PEO (Fig. 10(d)) has very asymmetric positive and negative lobes, whereas the (Newtonian) glycerol is approximately antisymmetric around the resonant frequency (Fig. 10(c)). The 1\% PEO solution has a finite crosscorrelation at zero time lag, whereas the glycerol has zero cross-correlation at zero time lag. For our apparatus zero time lag means $0-1 \mu \mathrm{s}$ time lag $\left(f_{\mathrm{Ny}}=500 \mathrm{kHz}\right)$.

Thus, the cross-correlation between two cantilevers has obvious, qualitative features that distinguish PEO from Newtonian liquids, whereas the auto-correlation of a single cantilever does not. In fact, the distinguishing features in the cross-correlation are related: the area under $G_{12}$ is proportional to the value of the cross-correlation at zero time lag,

TABLE II. Parameters for the fits of Eq. (3) to the data in Fig. 6.

\begin{tabular}{|c|c|c|c|c|c|c|c|c|}
\hline \multirow[b]{2}{*}{ (\% wt.) Glycerol solution } & \multicolumn{3}{|c|}{ Fit parameters } & \multicolumn{3}{|c|}{ Derived quantities } & \multirow[b]{2}{*}{$\gamma_{a \text {, theory }}[\mathrm{mg} / \mathrm{s}]$} & \multirow[b]{2}{*}{$\eta[\mathrm{mPa} \cdot \mathrm{s}]$} \\
\hline & $\gamma_{a}[\mathrm{mg} / \mathrm{s}]$ & $\gamma_{c}[\mathrm{mg} / \mathrm{s}]$ & $\omega_{r} / \omega_{o}$ & $m[\mathrm{ng}]$ & $\mathrm{m} / \mathrm{m}_{e}$ & $Q$ & & \\
\hline 16 & 1.68 & 0.310 & 0.32 & 35 & 3.7 & 2.9 & 2.18 & 1.53 \\
\hline 24 & 1.70 & 0.314 & 0.31 & 36 & 3.8 & 2.9 & 2.48 & 1.97 \\
\hline 44 & 2.31 & 0.361 & 0.30 & 39 & 4.1 & 2.3 & 3.56 & 3.77 \\
\hline 56 & 2.87 & 0.398 & 0.28 & 43 & 4.5 & 1.9 & 5.00 & 6.77 \\
\hline
\end{tabular}



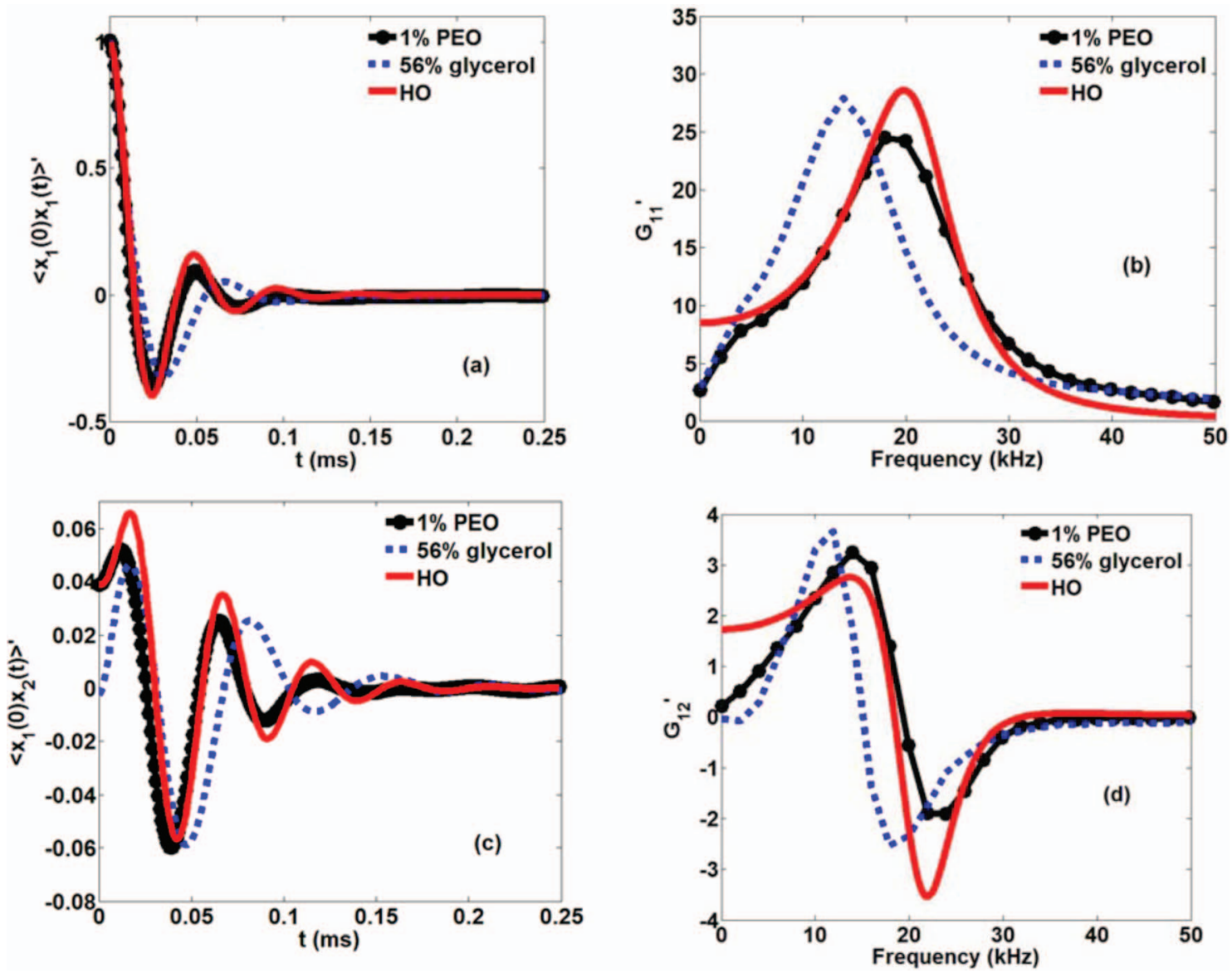

FIG. 10. (a) and (b) Auto-correlation and noise spectrum of equilibrium fluctuations in cantilever displacement for a single cantilever in $1 \%$ PEO and comparison with data in 56\% glycerol in water solution and HO model (Eq. (10)). The noise spectrum is calculated using Eq. (13) in Ref. 31. (c) and (d) Cross-correlation and noise spectrum for the same conditions as (a). The noise spectrum is calculated using Eq. (14) in Ref. 31. Note that (a) and (b) are measured in the presence of a second cantilever. The cantilever pair is ORC8-A (length $=100 \mu \mathrm{m}$, width $=40 \mu \mathrm{m}, k=0.71 \mathrm{~N} / \mathrm{m}$ ). Note that the non-Newtonian PEO solution has a non-zero cross-correlation at zero time and asymmetric lobes in the noise spectrum.

that is:

$$
\left\langle x_{1}(0) x_{2}(0)\right\rangle=\frac{2}{\pi} \int_{0}^{\infty} G_{12}(\omega) d \omega .
$$

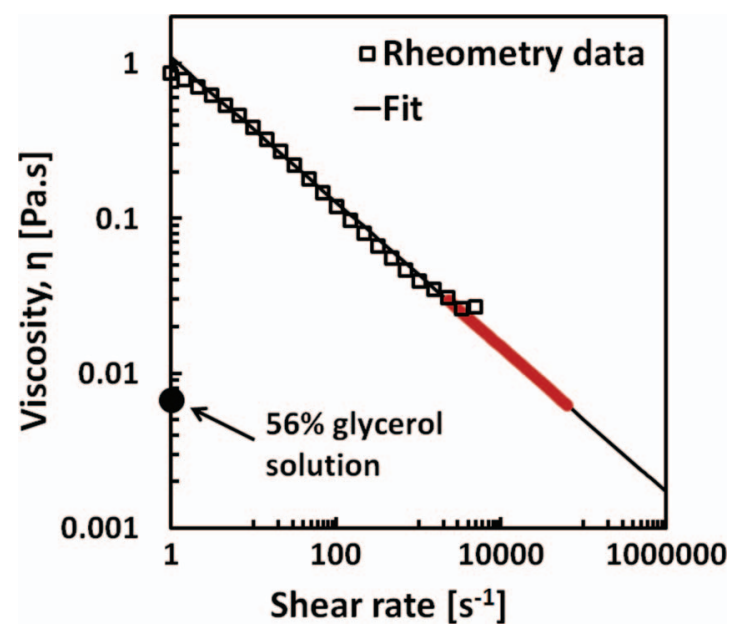

FIG. 11. Viscosity of PEO measured by a conventional controlled shear rheometer (square symbols). The black line is a power law fit to the data, extrapolated to higher shear rates. The fit parameters are $K=1.1$ and $n=0.53$ in power law expression $\eta=K \dot{\gamma}^{n-1}$, where $\dot{\gamma}$ is the shear rate. The solid red (thick) line shows the resonant frequency range of ORC8-A cantilever in $1 \%$ PEO.
The proof is provided in Appendix B.

The qualitative explanation for this asymmetry is that the shear thinning fluid has a larger response at lower frequency because of the greater viscosity, so the spectrum is asymmetric with a larger response at lower frequency. To become more quantitative, we utilize the harmonic oscillator model.

Conceptually, the easiest way to model the experimentally observed zero time lag cross-correlation is to add a spring (constant, $k_{\mathrm{fl}}$ ) between the two masses (see Fig. 12 schematic). This is a model for a Boger fluid. ${ }^{43}$ The resulting equations of motion are

$\ddot{X}_{1}+\left(\frac{\gamma_{a}}{m}+\frac{\gamma_{c}}{m}\right) \dot{X}_{1}+\frac{\gamma_{c}}{m} \dot{X}_{2}+\left(\omega_{r}^{2}+\frac{k_{\mathrm{fl}}}{m}\right) X_{1}-\frac{k_{\mathrm{fl}}}{m} X_{2}=0$,

$\ddot{X}_{2}+\left(\frac{\gamma_{a}}{m}+\frac{\gamma_{c}}{m}\right) \dot{X}_{2}+\frac{\gamma_{c}}{m} \dot{X}_{1}+\left(\omega_{r}^{2}+\frac{k_{\mathrm{fl}}}{m}\right) X_{2}-\frac{k_{\mathrm{fl}}}{m} X_{1}=0$.

The solution to Eq. (9) can again be expressed in terms of a symmetric and antisymmetric normal mode:

$$
\begin{aligned}
X_{s}= & \exp \left(-\omega_{d} t\right)\left(1+X_{2}(0)\right)\left[\cos \left(\sqrt{\omega_{r}^{2}-\omega_{d}^{2} t}\right)\right. \\
& \left.+\frac{\omega_{d}}{\sqrt{\omega_{r}^{2}-\omega_{d}^{2}}} \sin \left(\sqrt{\omega_{r}^{2}-\omega_{d}^{2}} t\right)\right],
\end{aligned}
$$




$$
X_{a}=\exp \left(-\frac{\gamma_{a}}{2 m} t\right)\left(1-X_{2}(0)\right)\left[\begin{array}{l}
\cos \left(\sqrt{\left(\omega_{r}^{2}+\frac{2 k_{\mathrm{ff}}}{m}\right)-\left(\frac{\gamma_{a}}{2 m}\right)^{2} t}\right)+ \\
\frac{\frac{\gamma_{a}}{2 m}}{\sqrt{\left(\omega_{r}^{2}+\frac{2 k_{\mathrm{fl}}}{m}\right)-\left(\frac{\gamma_{a}}{2 m}\right)^{2}}} \sin \left(\sqrt{\left(\omega_{r}^{2}+\frac{2 k_{\mathrm{fl}}}{m}\right)-\left(\frac{\gamma_{a}}{2 m}\right)^{2} t}\right)
\end{array}\right],
$$

where $X_{2}(0)=$ zero time lag cross-correlation and the displacement has again been normalized by the initial displacement to cantilever 1. Compared to Eqs. (6) and (7), the symmetric stretch is unchanged by the addition of the spring, but the antisymmetric stretch now depends on the stiffness of the fluid that couples the springs $\left(k_{\mathrm{ff}}\right)$.

We determined the least-squared fit of Eqs. (10) and (11) to the auto- and cross-correlation data for PEO in the range zero to $0.3 \mathrm{~ms}$. $\gamma_{a}$ was obtained from the auto-correlation. In principal, we could fit both $\gamma_{c}$ and $k_{\mathrm{fl}}$ to the cross-correlation. Rather than fit two parameters at the same time, we recognize that in this model, the only non-Newtonian property of the fluid is the elasticity. The damping is still Newtonian, so we assume that $\gamma_{c}$ and $\gamma_{a}$ are each linear functions of viscosity, and thus linear functions of each other. We use the glycerol data to obtain $\gamma_{c}$ from $\gamma_{a}$ and then only have one fit parameter for the cross-correlation. This calibration is reasonable as $1 \%$ PEO solution has approximately the same density as glycerol solutions.

Figure 10 compares the measured data to the $\mathrm{HO}$ fits as well as to data for the Newtonian fluid, $56 \%$ glycerol. The HO model was fit to the finite value of the zero time lag crosscorrelation, but this was enough to capture the most salient difference between PEO and glycerol. The Fourier transform of the cross-correlation (Fig. 10(d)) shows that the fit is not as good at low frequencies: the simple model of elasticity plus constant viscosity does not capture the behavior at low frequency. The fit parameters are shown in Table II.

A complex fluid can also change its rheological properties over time due to changes in structure, so the acquisition

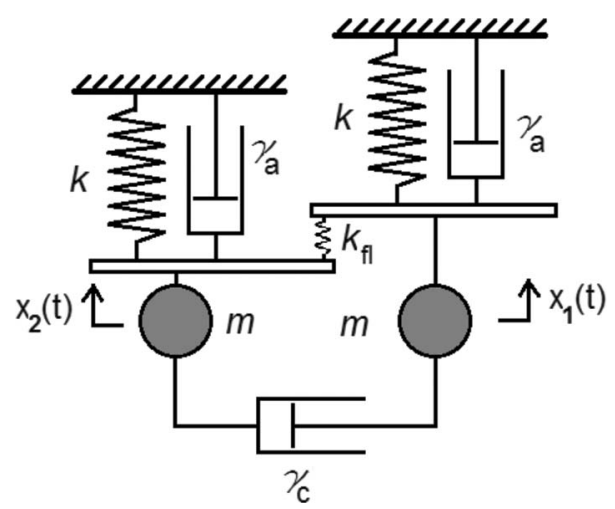

FIG. 12. Schematic of a cantilever pair modeled as a mass-spring-damper system in an elastic fluid. $k_{\mathrm{fl}}$ represents the elasticity of the intervening fluid. time of the correlation spectrum is important. The acquisition time is limited by the need to record each data bin for longer than the largest time lag required, and then to measure and average a number of such bins to reduce noise. For example, there is no significant difference in the data presented in Fig. 10 when the data bin is reduced from large times to $5 \mathrm{~ms}$, provided that 200 bins are averaged. Thus, the current time resolution for high fidelity monitoring of rheological changes in the range of $1-100 \mathrm{kHz}$ is about $1 \mathrm{~s}$.

\section{COMPARISON BETWEEN CFS AND MICRORHEOLOGY OR TRADITIONAL RHEOMETRY}

We note the following features of the CFS that are useful for rheological measurements. In contrast to the bead experiments of two-point microrheology, ${ }^{6,8,30}$ the cantilevers are fixed to the surroundings, and any number of solutions or suspensions can be washed across them for analysis. The mechanical properties can be optimized by changing the cantilever springs. The CFS can operate at very high speeds (MHz compared to $100 \mathrm{~Hz}$ ), ${ }^{6}$ measure very small deflections ( $<0.1 \mathrm{~nm}$ compared to $20 \mathrm{~nm}),{ }^{6}$ and with the use of nanoscopically curved tips can investigate the interactions between tiny amounts of material compared to two-point microrheology because the tips can be brought arbitrarily close to each other.

The variation in our results was negligible for changes in cantilever separation of several micrometers, and the expected reduction in the magnitude of the correlations was experimentally measured for large separations. In addition, numerical calculations showed that the cross-correlation was insensitive to vertical offsets between the cantilevers of several micrometers. The insensitivity to small variations in cantilever separation obviates the need for precise control of the cantilever during fabrication.

In comparison to traditional rheometry or current twopoint microrheometry, we note that the CFS can measure rheological properties over a broad range of frequencies simply by passively monitoring the motion of cantilevers in a remarkably simple device. The dimensions of the wet parts are only millimeters, so the device could be used as a dip probe, providing that either the fluid was reasonably transparent, or that non-optical (e.g., piezoelectric) cantilever sensing was used. The greatest disadvantage is that the shear stress is non-uniform, which complicates analysis. 
TABLE III. Parameters for the fits to PEO data.

\begin{tabular}{|c|c|c|c|c|c|c|}
\hline \multirow[b]{2}{*}{ Fluid } & \multicolumn{3}{|c|}{ Fit parameters } & \multicolumn{3}{|c|}{ Derived quantities } \\
\hline & $\gamma_{a}[\mathrm{mg} / \mathrm{s}]$ & $\omega_{r} / \omega_{o}$ & $k_{\mathrm{fl}}[\mathrm{mN} / \mathrm{m}]$ & $\gamma_{c}[\mathrm{mg} / \mathrm{s}]$ & $m[\mathrm{ng} / \mathrm{s}]$ & $Q$ \\
\hline $1 \%$ PEO solution & 2.7 & 0.29 & 31 & 0.387 & 40 & 2 \\
\hline
\end{tabular}

\section{APPLICATION OF CFS TO SINGLE MOLECULE STUDIES}

We now explore a second application for CFS. The twobead technique has been used to elucidate the motion of single molecules of DNA. ${ }^{36,44}$ The stiffness of the two-bead measurement system is generally weaker than the stiffness of the polymer, so the polymer is weakly perturbed. Owing to the greater stiffness of AFM cantilevers, most polymers will be pulled into non-linear response by the action of the thermal agitation of the cantilevers, so in many cases, the thermal noise measurements will be akin to a series of low-amplitude pulling experiments at a variety of amplitudes and frequencies.

The CFS benefits from the same advantages as the twobead cross-correlation measurements. For example, noise in the deflection detection system is greatly reduced in the crosscorrelation, provided that the noises in each system (e.g., from lasers and detectors) are not correlated. This is also true of the thermal noise: ${ }^{29}$ the two-bead experiment resulted in an improvement of force resolution of nearly an order of magnitude compared to the one-bead experiment. The measured correlations in Brownian force between the two cantilevers should also be much smaller than the Brownian force acting on a single cantilever. ${ }^{34}$ Figure 13 shows that indeed the noise in the cross-correlation is about $1 / 10$ of the noise in the autocorrelation for the same fluid, and thus that a fundamental noise limit in AFM single-molecule spectroscopy has been improved. ${ }^{32,34}$ The dispersion of noise in the cross-correlation also has the interesting feature that there is a particular frequency near the resonant frequency where the thermal noise is zero (see Fig. 13).

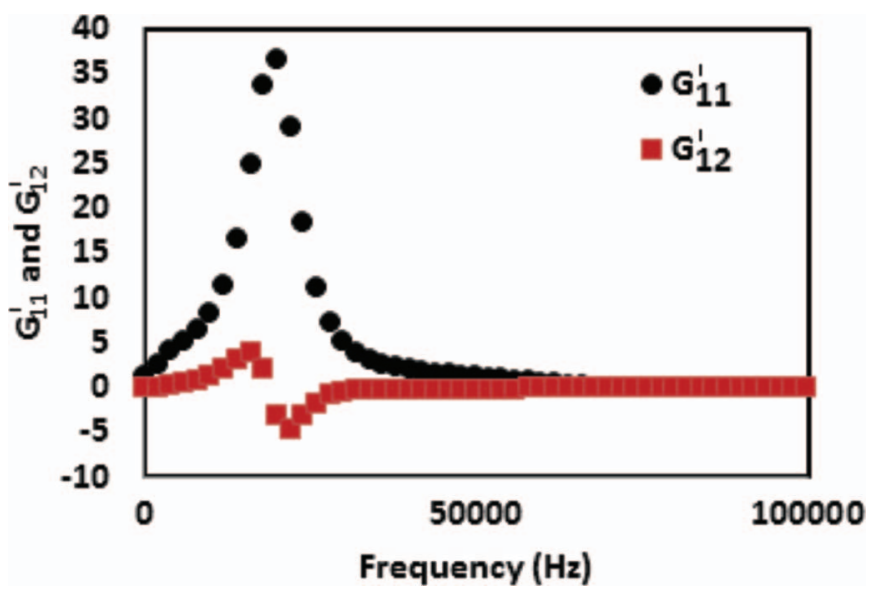

FIG. 13. Comparison between noise spectra of auto-correlation $\left(\mathrm{G}_{11}\right)$ and noise spectrum of cross-correlation between two cantilevers $\left(\mathrm{G}_{12}\right)$ in $16 \%$ glycerol solutions separated by $8 \mu \mathrm{m}$ at $23^{\circ} \mathrm{C}$ (ORC8 A: length $=100 \mu \mathrm{m}$, width $=40 \mu \mathrm{m}, k=0.71 \mathrm{~N} / \mathrm{m})$.
Another way of parameterizing the noise in the singlemolecule experiment is through the parameter fit in the $\mathrm{HO}$ model for $\gamma_{c}$. Note that $\gamma_{c}$ is about an order of magnitude smaller than the damping on the isolated cantilever, $\gamma_{a}$ (Tables I-III).

As described previously, ${ }^{31,32}$ the magnitude of the force noise, $F_{i j}$, can be estimated from the auto- and crosscorrelations:

$$
F_{i j}=k \sqrt{\left|\left\langle x_{i}(0) x_{j}(t)\right\rangle\right|_{\max }},
$$

where $i=j$ for the single cantilever measurement. The noise amplitude will depend in general on the separation between the cantilevers and the spring constant. For the experiment in water, the spring constant is $\sim 0.1 \mathrm{~N} / \mathrm{m}$ and the maximum amplitude of the cross-correlation is $\sim 0.0015 \mathrm{~nm}^{2}$, so $F_{\text {noise }}$ $\sim 4 \mathrm{pN}$ when the cantilevers are separated by $8 \mu \mathrm{m}$. This is a reasonable separation for two cantilevers separated by the height of two tips.

We also note that the use of two tips in CFS compared to one tip in conventional single-molecule force spectroscopy greatly reduces the van der Waals force acting the tip. This is significant because the van der Waals forces acting on the tip causes two problems in single-molecule work: adhesion of the tip to other solids and the introduction of a force that varies strongly with separation. For small separations, this spatially varying force must be subtracted from force measurements to obtain the single-molecule force.

Data from Fig. 10 also give us an estimate of the expected stiffness resolution of CFS for single-molecule studies. The fitted stiffness of the polymer solution was $31 \mathrm{mN} / \mathrm{m}$ for $1 \%$ PEO. This is the same order of magnitude stiffness found during single-molecule AFM experiments on poly(acrylic acid) and poly(vinyl alcohol). ${ }^{45,46}$ The resolution was obtained for a stiff cantilever $(k=0.71 \mathrm{~N} / \mathrm{m})$. Note that, for the HO model, the stiffness is directly proportional to the zero time lag intercept in the cross-correlation.

\section{CONCLUSIONS}

The first instrument that measures the cross-correlation in the vibrations of two closely spaced cantilevers in fluid has been built. The thermal fluctuations of these cantilevers are accurately described using the fluctuation-dissipation theorem. The stochastic dynamics of the cantilevers have been shown to depend upon the viscosity of the fluid so that the CFS can be used as a rheometer.

CFS is an experimentally simple approach to rheological measurements that is minimally invasive, can operate over a large range of frequencies and a large range of fluid elasticity, and can explore spatial variation of fluid properties. The device has no "moving" parts; it simply executes miniscule 
vibrations that are thermally driven, yet it can determine the frequency-dependent viscosity over a very broad frequency range (DC-MHz). The use of two probes rather than one probe is better for analyzing heterogeneous fluids, as described for two-particle microrheology.

A harmonic oscillator model, modified to include damping between the cantilevers, provides a good fit to the experimental data for Newtonian Fluids. This provides a fast and simple way to extract meaningful quantities from the experimental measurements. If two calibration liquids of known viscosity are measured, then the harmonic oscillator model can be used for rheometry. The addition of a spring between the cantilevers in the harmonic oscillator model captures some of the behavior of PEO solutions. The model can be used to measure the stiffness of an elastic fluid and demonstrates resolution suitable for measuring the effective spring constant of single molecules.

Thermal noise sets a fundamental limit to AFM singlemolecule force resolution. We have demonstrated that the CFS has a much lower noise floor than single cantilever measurements. The decreased noise arises because the noise along the length of each cantilever is not correlated; only the noise arising from fluid motion in the gap between the cantilevers is correlated.

\section{ACKNOWLEDGMENTS}

The work described in this paper was funded by the National Science Foundation via Award Number CBET-0959228 and by Virginia Tech.

\section{APPENDIX A}

The equations of motion for the system shown in Fig. 12 are

$$
\begin{aligned}
& \ddot{X}_{1}+\left(\frac{\omega_{r}}{Q}+\frac{\gamma_{c}}{m}\right) \dot{X}_{1}+\frac{\gamma_{c}}{m} \dot{X}_{2}+\left(\omega_{r}^{2}+\frac{k_{\mathrm{fl}}}{m}\right) X_{1}-\frac{k_{\mathrm{fl}}}{m} X_{2}=0, \\
& \ddot{X}_{2}+\left(\frac{\omega_{r}}{Q}+\frac{\gamma_{c}}{m}\right) \dot{X}_{2}+\frac{\gamma_{c}}{m} \dot{X}_{1}+\left(\omega_{r}^{2}+\frac{k_{\mathrm{fl}}}{m}\right) X_{2}-\frac{k_{\mathrm{fl}}}{m} X_{1}=0 .
\end{aligned}
$$

For the system in Fig. $5, k_{\mathrm{fl}}=0$.

We initially define two new coordinate systems:

$$
\begin{aligned}
& X_{s}=X_{1}+X_{2}, \\
& X_{a}=X_{1}-X_{2} .
\end{aligned}
$$

$X_{\mathrm{s}}$ is the mode where the vibrations are in phase (the symmetric vibration) and $X_{\mathrm{a}}$ is the mode when the vibrations are out of phase (the antisymmetric stretch). The equations of motion in the new coordinate system are

$$
\begin{aligned}
& \ddot{X}_{s}+\left(\frac{\gamma_{a}}{m}+\frac{2 \gamma_{c}}{m}\right) \dot{X}_{s}+\omega_{r}^{2} X_{s}=0, \\
& \ddot{X}_{a}+\frac{\gamma_{a}}{m} \dot{X}_{a}+\left(\omega_{r}^{2}+\frac{2 k_{\mathrm{ff}}}{m}\right) X_{a}=0,
\end{aligned}
$$

and the solution is

$$
\begin{aligned}
& X_{s}=\frac{-\left(1+X_{2}(0)\right)}{\left(n_{+x}-n_{-x}\right)}\left(n_{-x} \exp \left(n_{+x} t\right)-n_{+x} \exp \left(n_{-x} t\right)\right), \\
& X_{a}=\frac{-\left(1-X_{2}(0)\right)}{\left(n_{+y}-n_{-y}\right)}\left(n_{-y} \exp \left(n_{+y} t\right)-n_{+y} \exp \left(n_{-y} t\right)\right) .
\end{aligned}
$$

Expressed in terms of the deflections, the solution is

$$
\left\{\begin{aligned}
X_{1}= & \frac{-\left(1+X_{2}(0)\right)}{2\left(n_{+x}-n_{-x}\right)}\left[n_{-x} \exp \left(n_{+x} t\right)-n_{+x} \exp \left(n_{-x} t\right)\right] \\
& +\frac{-\left(1-X_{2}(0)\right)}{2\left(n_{+y}-n_{-y}\right)}\left[n_{-y} \exp \left(n_{+y} t\right)-n_{+y} \exp \left(n_{-y} t\right)\right], \\
X_{2}= & \frac{-\left(1+X_{2}(0)\right)}{2\left(n_{+x}-n_{-x}\right)}\left[n_{-x} \exp \left(n_{+x} t\right)-n_{+x} \exp \left(n_{-x} t\right)\right] \\
& +\frac{\left(1-X_{2}(0)\right)}{2\left(n_{+y}-n_{-y}\right)}\left[n_{-y} \exp \left(n_{+y} t\right)-n_{+y} \exp \left(n_{-y} t\right)\right],
\end{aligned}\right.
$$

where $X_{2}(0)$ is the deflection of cantilever 2 at zero time and the displacements have been normalized by $X_{1}(0)$. In the limit of linear response, $X_{2}(0)$ can be obtained from the zero time lag cross-correlation, which in Newtonian fluids is zero. The rest of the coefficients are as follows:

$$
\begin{aligned}
& n_{+x,-x}=\frac{-c_{x}^{*} \pm \sqrt{c_{x}^{* 2}-4 k_{x}^{*}}}{2}, \\
& n_{+y,-y}=\frac{-c_{y}^{*} \pm \sqrt{c_{y}^{* 2}-4 k_{y}^{*}}}{2},
\end{aligned}
$$

where

$$
\begin{aligned}
c_{x}^{*} & =\frac{\gamma_{a}}{m}+\frac{2 \gamma_{c}}{m}, \\
c_{y}^{*} & =\frac{\gamma_{a}}{m}, \\
k_{x}^{*} & =\omega_{r}^{2}, \\
k_{y}^{*} & =\omega_{r}^{2}+\frac{2 k_{\mathrm{fl}}}{m} .
\end{aligned}
$$

Inserting the above expressions into the expression for $X_{1}$ and $X_{2}$ results in Eqs. (6), (7), (10), and (11). The noise spectrum can be obtained using Eqs. (13) and (14) in Ref. 31 as follows:

$G_{11}(\omega)$

$$
\begin{aligned}
= & \left(\frac{4 \omega_{d} \omega_{r}^{2}\left(1+X_{2}(0)\right)}{\omega^{4}-2\left(\omega_{r}^{2}-2 \omega_{d}^{2}\right) \omega^{2}+\omega_{r}^{4}}\right) \\
& +\left(\frac{2\left(\frac{\gamma_{a}}{m}\right)\left(\omega_{r}^{2}+\frac{2 k_{\mathrm{fl}}}{m}\right)\left(1-X_{2}(0)\right)}{\omega^{4}-2\left(\omega_{r}^{2}+\frac{2 k_{\mathrm{fl}}}{m}-\frac{1}{2}\left(\frac{\gamma_{a}}{m}\right)^{2}\right) \omega^{2}+\left(\omega_{r}^{2}+\frac{2 k_{\mathrm{fl}}}{m}\right)^{2}}\right),
\end{aligned}
$$




$$
\begin{aligned}
G_{12}(\omega) & \\
= & \left(\frac{4 \omega_{d} \omega_{r}^{2}\left(1+X_{2}(0)\right)}{\omega^{4}-2\left(\omega_{r}^{2}-2 \omega_{d}^{2}\right) \omega^{2}+\omega_{r}^{4}}\right) \\
& -\left(\frac{2\left(\frac{\gamma_{a}}{m}\right)\left(\omega_{r}^{2}+\frac{2 k_{\mathrm{fl}}}{m}\right)\left(1-X_{2}(0)\right)}{\omega^{4}-2\left(\omega_{r}^{2}+\frac{2 k_{\mathrm{fl}}}{m}-\frac{1}{2}\left(\frac{\gamma_{a}}{m}\right)^{2}\right) \omega^{2}+\left(\omega_{r}^{2}+\frac{2 k_{\mathrm{fl}}}{m}\right)^{2}}\right) .
\end{aligned}
$$

\section{APPENDIX B}

From the definition of $G_{12}$ (Eq. (14) in Ref. 31), we have

$$
G_{12}(\omega)=4 \int_{0}^{\infty}\left\langle x_{1}(0) x_{2}(t)\right\rangle \cos (\omega t) d t .
$$

The inverse of this Fourier transform is

$$
\left\langle x_{1}(0) x_{2}(t)\right\rangle=\frac{2}{\pi} \int_{0}^{\infty} G_{12}(\omega) \cos (\omega t) d \omega \text {. }
$$

At zero time lag $(t=0)$, the above equation simplifies to

$$
\left\langle x_{1}(0) x_{2}(0)\right\rangle=\frac{2}{\pi} \int_{0}^{\infty} G_{12}(\omega) d \omega,
$$

which is $\frac{2}{\pi}$ times the area under $G_{12}(\omega)$.

${ }^{1}$ R. G. Larson, The Structure and Rheology of Complex Fluids (Oxford University Press, New York, 1998).

${ }^{2}$ M. L. Gardel, M. T. Valentine, and D. A. Weitz, Microscale Diagnostic Techniques (Springer-Verlag, 2005).

${ }^{3}$ F. Ziemann, J. Radler, and E. Sackmann, Biophys. J. 66(6), 2210-2216 (1994).

${ }^{4}$ T. G. Mason and D. A. Weitz, Phys. Rev. Lett. 75(14), 2770-2773 (1995).

${ }^{5}$ T. G. Mason, K. Ganesan, J. H. vanZanten, D. Wirtz, and S. C. Kuo, Phys. Rev. Lett. 79(17), 3282-3285 (1997).

${ }^{6}$ J. C. Crocker, M. T. Valentine, E. R. Weeks, T. Gisler, P. D. Kaplan, A. G. Yodh, and D. A. Weitz, Phys. Rev. Lett. 85(4), 888-891 (2000).

${ }^{7}$ R. R. Brau, J. M. Ferrer, H. Lee, C. E. Castro, B. K. Tam, P. B. Tarsa, P. Matsudaira, M. C. Boyce, R. D. Kamm, and M. J. Lang, J. Opt. A, Pure Appl. Opt. 9(8), S103-S112 (2007).

${ }^{8}$ T. M. Squires and T. G. Mason, Annu. Rev. Fluid Mech. 24, 413-438 (2010).

${ }^{9}$ D. T. Chen, E. R. Weeks, J. C. Crocker, M. F. Islam, R. Verma, J. Gruber, A. J. Levine, T. C. Lubensky, and A. G. Yodh, Phys. Rev. Lett. 90(10), $108301(2003)$
${ }^{10}$ F. Gittes, B. Schnurr, P. D. Olmsted, F. C. MacKintosh, and C. F. Schmidt, Phys. Rev. Lett. 79(17), 3286-3289 (1997).

${ }^{11}$ M. Buchanan, M. Atakhorrami, J. F. Palierne, and C. F. Schmidt, Macromolecules 38(21), 8840-8844 (2005).

${ }^{12}$ F. Cardinaux, L. Cipelletti, F. Scheffold, and P. Schurtenberger, Europhys. Lett. 57(5), 738-744 (2002)

${ }^{13}$ B. Schnurr, F. Gittes, F. C. MacKintosh, and C. F. Schmidt, Macromolecules 30(25), 7781-7792 (1997).

${ }^{14}$ J. Liu, M. L. Gardel, K. Kroy, E. Frey, B. D. Hoffman, J. C. Crocker, A. R. Bausch, and D. A. Weitz, Phys. Rev. Lett. 96(11), 118104 (2006).

${ }^{15}$ S. Boskovic, J. W. M. Chon, P. Mulvaney, and J. E. Sader, J. Rheol. 46(4), 891-899 (2002).

${ }^{16}$ M. Hennemeyer, S. Burghardt, and R. W. Stark, Sensors 8(1), 10-22 (2008).

${ }^{17}$ M. Youssry, N. Belmiloud, B. Caillard, C. Ayela, C. Pellet, and I. Dufour, Sens. Actuators, A 172, 40-46 (2011).

${ }^{18}$ R. Motamedi and P. M. Wood-Adams, J. Rheol. 54(5), 959-980 (2010).

${ }^{19}$ S. Basak and A. Raman, Phys. Fluids 19(1), 017105 (2007).

${ }^{20}$ S. Basak, A. Raman, and S. V. Garimella, J. Appl. Phys. 99(11), 114906 (2006).

${ }^{21}$ J. W. M. Chon, P. Mulvaney, and J. E. Sader, J. Appl. Phys. 87(8), 39783988 (2000).

${ }^{22}$ A. M. Korsunsky, S. Cherian, R. Raiteri, and R. Berger, Sens. Actuators, A 139(1-2), 70-77 (2007).

${ }^{23}$ A. Raman, J. Melcher, and R. Tung, Nanotoday 3(1), 20-27 (2008).

${ }^{24}$ J. E. Sader, J. Appl. Phys. 84(1), 64-76 (1998).

${ }^{25}$ M. Spletzer, A. Raman, H. Sumali, and J. P. Sullivan, Appl. Phys. Lett. 92(11), 114102 (2008).

${ }^{26}$ R. W. Stark, T. Drobek, and W. M. Heckl, Ultramicroscopy 86(1-2), 207215 (2001).

${ }^{27}$ C. A. V. Eysden and J. E. Sader, J. Appl. Phys. 101(4), 044908 (2007).

${ }^{28}$ S. Kim, K. D. Kihm, and T. Thundat, Exp. Fluids 48, 721-736 (2010).

${ }^{29}$ J. C. Meiners and S. R. Quake, Phys. Rev. Lett. 82(10), 2211-2214 (1999).

${ }^{30}$ T. A. Waigh, Rep. Prog. Phys. 68(3), 685-742 (2005).

${ }^{31}$ M. R. Paul, M. T. Clark, and M. C. Cross, Nanotechnology 17(17), 45024513 (2006).

${ }^{32}$ M. T. Clark and M. R. Paul, Int. J. Non-Linear Mech. 42(4), 690-696 (2007).

${ }^{33}$ R. W. Stark, Nanotechnology 15, 347-351 (2004).

${ }^{34}$ J. L. Arlett, M. R. Paul, J. Solomon, M. C. Cross, S. E. Fraser, and M. L. Roukes, Lect. Notes Phys. 711, 241-270 (2007).

${ }^{35}$ M. R. Paul and M. C. Cross, Phys. Rev. Lett. 92(23), 235501 (2004).

${ }^{36}$ J. C. Meiners and S. R. Quake, Phys. Rev. Lett. 84(21), 5014-5017 (2000).

${ }^{37}$ G. Meyer and N. M. Amer, Appl. Phys. Lett. 53, 1045-1047 (1988)

${ }^{38}$ J. L. Hutter and J. Bechhoefer, Rev. Sci. Instrum. 64, 1868-1873 (1993).

${ }^{39}$ C. D. F. Honig, M. Radiom, B. A. Robbins, J. Y. Walz, M. R. Paul, and W. A. Ducker, Appl. Phys. Lett. 100(5), 053121 (2012).

${ }^{40}$ ESI Group, 2012, see http://www.esi-cfd.com/.

${ }^{41}$ R. J. Clarke, O. E. Jensen, J. Billingham, A. P. Pearson, and P. M. Williams, Phys. Rev. Lett. 96, 050801 (2006).

${ }^{42}$ H.-J. Butt and M. Jaschke, Nanotechnology 6, 1-7 (1995).

${ }^{43}$ D. F. James, Annu. Rev. Fluid Mech. 41, 129-142 (2009).

${ }^{44}$ S. R. Quake, H. Babcock, and S. Chu, Nature 388(6638), 151-154 (1997).

${ }^{45}$ H. B. Li, B. B. Liu, X. Zhang, C. X. Gao, J. C. Shen, and G. T. Zou, Langmuir 15(6), 2120-2124 (1999).

${ }^{46}$ H. B. Li, W. K. Zhang, W. Q. Xu, and X. Zhang, Macromolecules 33(2), 465-469 (2000) 